\title{
NOUVELle
}

\section{Aspergillose invasive}

\section{Perspectives en infectiologie préventive}

Frédéric Lamoth, Pierre-yves Bochud
Institut de microbiologie, Service

des maladies infectieuses, département de médecine, Centre hospitalier universitaire Vaudois (CHUV) et Université de Lausanne, Rue du Bugnon 48, 1011 Lausanne, Suisse. Pierre-Yves.Bochud@chuv.ch
> Durant la dernière décennie, notre compréhension du système immunitaire inné a considérablement bénéficié de la découverte d'une série de «détecteurs de motifs moléculaires » microbiens (pattern-recognition receptors, PRR) qui sont à l'interface de l'hôte et du pathogène [23]. Combinée au développement de la génétique des populations et à des techniques de génotypage toujours plus performantes, cette découverte pourrait permettre une meilleure prévention par la détermination du risque infectieux chez les patients dont le système immunitaire est altéré. Des études récentes suggèrent l'intérêt d'une telle approche dans la prévention de l'aspergillose invasive chez les patients subissant une greffe allogénique de cellules souches hématopoïétiques.

\section{Aspergillose invasive}

Aspergillus fumigatus est un champignon filamenteux ubiquitaire dans l'air et dans l'environnement qui peut provoquer des infections localisées ou disséminées chez l'être humain porteur d'un déficit de l'immunité. Après inhalation sous forme de conidies (spores), A. fumigatus a la capacité de germiner en hyphes (filaments composés de plusieurs cellules formant un réseau ramifié) et d'envahir le parenchyme pulmonaire. L'aspergillose invasive est une complication particulièrement redoutée durant les phases de neutropénie prolongée faisant suite aux chimiothérapies myéloablatives pour leucémie aiguë (elle atteint 5 à $8 \%$ des patients) ou après transplantation de moelle osseuse allogénique (12\% des patients).
Malgré le développement de nouvelles molécules antifongiques, cette infection reste associée à un haut taux de mortalité (40 à $70 \%$ ). L'initiation précoce d'un traitement adéquat constitue l'une des clés du succès thérapeutique. Cet objectif est souvent difficile à atteindre en raison de la sensibilité limitée des tests diagnostiques et du manque de spécificité des signes cliniques. Les stratégies de prise en charge de l'aspergillose invasive se basent sur une approche prophylactique ou empirique consistant à traiter un grand nombre de patients pour prévenir la survenue d'un seul épisode avec des conséquences potentiellement néfastes (coûts, risque de toxicité, développement de résistances). Il est donc nécessaire d'améliorer ces stratégies préventives, tant sur le plan diagnostique que thérapeutique.

\section{Immunité innée : première réponse} à l'entrée du pathogène

Le système immunitaire inné constitue la première ligne de défense de l'organisme contre les infections. Les agents pathogènes qui franchissent les barrières de protection naturelle entrent en interaction avec des cellules sentinelles (par exemple les macrophages, les monocytes ou les cellules dendritiques) dotées de récepteurs spécialisés (PRR) dans la détection de motifs moléculaires microbiens (microbe-associated molecular patterns, MAMP) [1]. Ces récepteurs assurent l'interface moléculaire entre l'hôte et le pathogène en initiant les mécanismes de la réponse immunitaire innée (production de cytokines et de chimiokines), puis de la réponse adaptative. Ils peuvent être classifiés en deux groupes principaux selon leur localisation cellulaire. Les récepteurs transmembranaires sont principalement représentés par la famille des récepteurs Toll-like (TLR), et par certains membres de la famille des lectines de type $C$ (Dectin-1). Les récepteurs intracytoplasmiques comprennent les récepteurs NOD-like (NLR, nucleotide-binding oligomerization domain (NOD)-like receptors) et RIG-I like (retinoic-acid-inducible protein I (RIG-I)-like receptors (RLR) [1].

La famille des TLR comprend au moins une dizaine de récepteurs chez les vertébrés [23]. Certains TLR sont préférentiellement localisés à la surface des cellules et détectent différents motifs microbiens, comme le lipopolysaccharide présent dans la membrane des bactéries à Gram-négatif (TLR4), la flagelline, composant principal des flagelles (TLR5) ou encore certains types de lipopeptides bactériens (TLR2 en coopération avec TLRl ou TLR6). D'autres TLR (TLR3, TLR7, TLR8 et TLR9) sont localisés dans des vésicules endocytaires et détectent divers types d'acides nucléiques [1]. Les récepteurs cytoplasmiques NODl et NOD2 et la famille des protéines NALP sont regroupés sous le terme de NLR (NOD-like or NACHT-LRR containing receptors). NOD1 et NOD2 détectent le peptidoglycane des parois bactériennes. NALP3, un des NALP les mieux connus, est activé par des composants exogènes ou endogènes [24], tels que I'ATP, I'ARN bactérien, l'acide urique et l'alum. Les RLR comprennent deux molécules (RIG-1 et MDA-5) impliquées dans la production d'interférons de type I en réponse aux infections virales [1]. 
Diverses études ont démontré que la détection de $A$. fumigatus implique plusieurs récepteurs de motif moléculaires, comprenant TLR2, Dectin-1, TLR4 et possiblement TLR9 [2-5]. D'autres produits de gènes plus ou moins indépendants $d u$ système TLR tels que la MAPK (mitogen activated protein kinase), la pentraxine 3 (PTX3), des protéines du surfactant (SP-A, SP-D) et des mannose-binding lectins ( $\mathrm{MBL}$ ) sont également impliqués dans la réponse immunitaire à $A$. fumigatus [6-9]. La mise en jeu des différents récepteurs est vraisemblablement influencée par les variations de l'état cellulaire du pathogène au cours des différentes phases de l'infection (conidies ou hyphes, impliquant l'exposition de différents composants cellulaires au système immunitaire) [10] et de l'état d'immunosuppression du patient (neu- tropénie, type de traitements immunosuppresseurs, maladies sous-jacentes) [11].

\section{Immunogénétique}

du système immunitaire inné

L'importance du système TLR de détection des pathogènes est illustrée par l'existence d'immunodéficiences primaires causées par des mutations affectant les molécules de leurs voies de transduction (par exemple MyD88, IRAK4, IKK $\gamma, I \kappa B \alpha)$. Elles ont été décrites pour la plupart par Jean-Laurent Casanova et ses collaborateurs au cours de la dernière décennie [12] (Figure 1). II s'agit de maladies rares entraînant généralement des infections répétées, souvent dues à des germes pyogènes, chez les enfants affectés (hérédité monogénique). La sévérité des phé- notypes suggère que les molécules relayant la transduction du système TLR sont essentielles pour la détection des pathogènes et leurs fonctions peu redondantes.

Ces observations contrastent avec les polymorphismes des gènes TLR euxmêmes qui sont relativement fréquents dans la population générale et semblent entraîner, à quelques exceptions près, des modifications moins drastiques de la réponse immunitaire (hérédité polygénique). En fait, il existe une profusion d'études rapportant des associations entre des polymorphismes des TLR (le plus souvent des polymorphismes touchant un nucléotide unique, ou SNP (single nucleotide polymorphism) et la susceptibilité à certaines infections ou à d'autres maladies [1, 13]. La qualité de ces études est diverse, souvent limitée

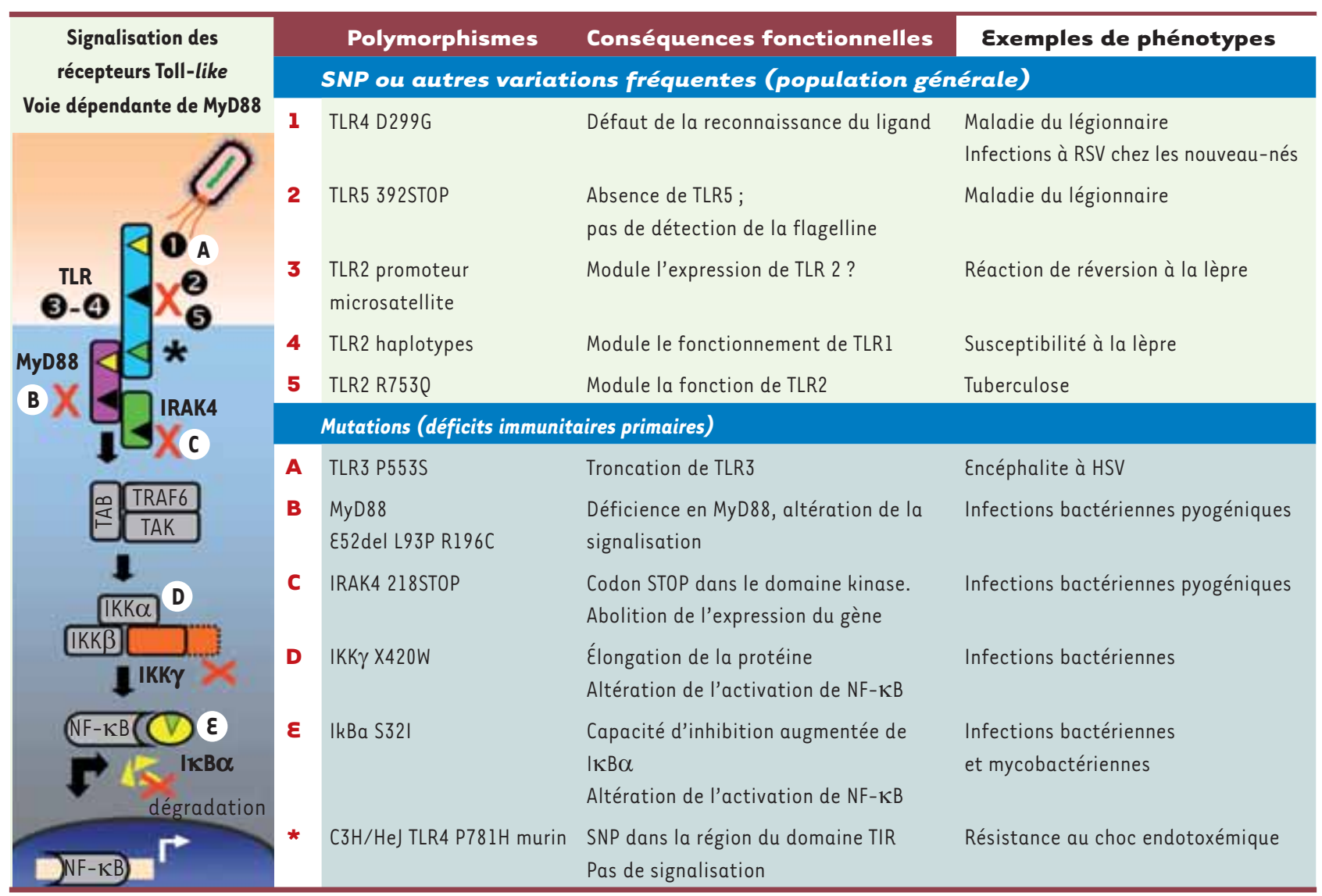

Figure 1. Exemples de variations génétiques qui modulent la réponse immune de l'hôte. Les exemples concernent des gènes/polymorphismes humains (sauf indications contraires). RSV : respiratory syncytial virus; HSV : Herpes simplex virus ; IRAK4 : interleukin-1 receptor-associated kinase 4 ; TIR domain : Toll/Interleukin-1 receptor (adaptée de [1]). 


\begin{tabular}{|c|c|c|c|c|c|c|}
\hline $\begin{array}{l}\text { Référence } \\
\text { (auteur, } \\
\text { année) }\end{array}$ & Population & $\begin{array}{l}\text { Gènes } \\
\text { impliqués }\end{array}$ & Nucléotide & $\begin{array}{l}\text { Acide } \\
\text { aminé }\end{array}$ & Commentaires & Association \\
\hline $\begin{array}{l}\text { Kesh et al. } \\
2005[17]\end{array}$ & $\begin{array}{l}\text { Greffe de moelle allogénique } \\
\text { Aspergillose invasive }\end{array}$ & $\begin{array}{l}\text { TLRI } \\
\text { TLRI et } \\
\text { TLR6 }\end{array}$ & $\begin{array}{l}239 \mathrm{G} / \mathrm{C} \\
743 \mathrm{~A} / \mathrm{G} \text { et } \\
745 \mathrm{C} / \mathrm{T}\end{array}$ & $\begin{array}{l}\text { R80T } \\
\text { N248S et } \\
\text { S249P }\end{array}$ & $\begin{array}{l}\text { Petit collectif } \\
\text { Pas de validation }\end{array}$ & Risque augmenté \\
\hline $\begin{array}{l}\text { Bochud et al. } \\
2008[16]\end{array}$ & $\begin{array}{l}\text { Greffe de moelle allogénique } \\
\text { Aspergillose invasive }\end{array}$ & TLR4 & $\begin{array}{l}1063 \mathrm{~A} / \mathrm{G} \\
1363 \mathrm{C} / \mathrm{T}\end{array}$ & $\begin{array}{l}\mathrm{D} 299 \mathrm{G} \\
\mathrm{T} 3991\end{array}$ & $\begin{array}{l}\text { Grand collectif }{ }^{2} \\
\text { Validation }{ }^{3}\end{array}$ & Risque augmenté \\
\hline $\begin{array}{l}\text { Seo et al. } \\
2005[20]\end{array}$ & $\begin{array}{l}\text { Greffe de moelle allogénique } \\
\text { Aspergillose invasive }\end{array}$ & IL-10 & $\begin{array}{l}819 \mathrm{C} / \mathrm{T} \\
592 \mathrm{C} / \mathrm{A}\end{array}$ & $\begin{array}{l}\text { aucun } \\
\text { aucun }\end{array}$ & $\begin{array}{l}\text { Ethnicité asiatique } \\
\text { Petit collectif } \\
\text { Pas de validation }\end{array}$ & Risque diminué \\
\hline $\begin{array}{l}\text { Sainz et al. } \\
2007 \text { [18] }\end{array}$ & $\begin{array}{l}\text { Hémopathies malignes } \\
\text { Aspergillose invasive }\end{array}$ & IL-10 & $1082 \mathrm{~A} / \mathrm{A}$ & aucun & $\begin{array}{l}\text { Petit collectif } \\
\text { Pas de validation }\end{array}$ & Risque diminué \\
\hline $\begin{array}{l}\text { Sainz et al. } \\
2007 \text { [19] }\end{array}$ & $\begin{array}{l}\text { Hémopathies malignes } \\
\text { Aspergillose invasive }\end{array}$ & TNFR2 & VNTR (-322) & aucun & $\begin{array}{l}\text { Petit collectif } \\
\text { Pas de validation }\end{array}$ & Risque augmenté \\
\hline $\begin{array}{l}\text { Crosdale et al. } \\
2001 \text { [21] }\end{array}$ & $\begin{array}{l}\text { Non immunodéprimés } \\
\text { Aspergillose pulmonaire }\end{array}$ & $M B L$ & $\mathrm{C} 868 \mathrm{~T}$ & C52R & $\begin{array}{l}\text { Petit collectif } \\
\text { Pas de validation }\end{array}$ & Risque augmenté \\
\hline $\begin{array}{l}\text { Vaid et al. } \\
2007 \text { [22] }\end{array}$ & $\begin{array}{l}\text { Non immunodéprimés } \\
\text { Aspergillose pulmonaire }\end{array}$ & $M B L$ & C868T & C52R & $\begin{array}{l}\text { Petit collectif } \\
\text { Pas de validation }\end{array}$ & Risque augmenté \\
\hline \multirow[t]{2}{*}{$\begin{array}{l}\text { Carvalho et al. } \\
2008 \text { [15] }\end{array}$} & $\begin{array}{l}\text { Non immunodéprimés } \\
\text { Aspergillose pulmonaire }\end{array}$ & TLR4 & $1063 \mathrm{~A} / \mathrm{G}$ & D299G & $\begin{array}{l}\text { Petit collectif } \\
\text { Pas de validation }\end{array}$ & Risque augmenté \\
\hline & $\begin{array}{l}\text { Non immunodéprimés } \\
\text { Aspergillose broncho- } \\
\text { pulmonaire allergique }\end{array}$ & TLR9 & $-1237 \mathrm{~T} / \mathrm{C}$ & aucun & $\begin{array}{l}\text { Petit collectif } \\
\text { Pas de validation }\end{array}$ & Risque augmenté \\
\hline
\end{tabular}

Tableau I. Association entre la présence de polymorphismes de médiateurs de l'immunité innée et le risque d'aspergillose. TLR: récepteur toll-like ; IL = interleukine ; TNF : tumor necrosis factor ; LT : lymphotoxine ; TNFR : récepteur du TNF ; VNTR : variable nucleotide tandem repeats ; MBL : mannose binding lectin. ${ }^{1}$ Petit collectif: $<200$ patients. ${ }^{2}$ Grand collectif : > 200 patients. ${ }^{3}$ Résultats confirmés dans un groupe de validation dans la même publication.

par l'absence de prise en compte de facteurs confondants (ethnicité, contrôles non exposés au même risque que les cas étudiés, définition du phénotype), des méthodes statistiques inappropriées (interprétation ne tenant pas compte de la puissance de l'étude, taille d'échantillon insuffisante, absence de correction pour tests multiples, non prise en compte de facteurs de risque non génétiques) et l'absence de tests fonctionnels pour soutenir les hypothèses biologiques. Quelques polymorphismes semblent toutefois exercer un rôle réel, parce que leur association avec des phénotypes clairs est répétée ou que leurs conséquences fonctionnelles sont démontrées [1, 13].
Perspectives pour une stratégie préventive de l'aspergillose invasive Deux polymorphismes de TLR4 (896 A/G [D299G] et 1196 C/T [T399I]), pratiquement toujours trouvés en paire chez les Caucasiens (fréquence des allèles environ $7 \%$ ) pourraient influencer la susceptibilité à différentes formes cliniques d'aspergillose. Ces deux polymorphismes semblent affecter la fonction de TLR4, bien que ces observations restent controversées [14]. Chez des individus sans immunosuppression notable, les allèles $299 \mathrm{G}$ et 3991 ont été associés à la survenue d'aspergillose chronique cavitaire [15]. Chez des individus subissant une greffe allogénique de cellules souches hématopoiétiques, ils ont été associés à la survenue d'aspergillose pulmonaire invasive [16]. La dernière observation a été confirmée dans une étude de validation. Les receveurs de greffe allogénique ont la particularité de posséder un système immunitaire «chimérique » dérivant en partie du receveur (cellules épithéliales) et en partie du donneur (cellules d'origine hématopoïétique). Les deux polymorphismes associés à l'aspergillose invasive dans cette population provenaient du donneur et non du receveur. La présence des allèles $299 \mathrm{G}$ et 3991 chez le donneur et/ou d'une séropositivité à CMV (cytomégalovirus) - du donneur ou du receveur - était associée à un risque plus élevé d'aspergillose invasive (12\% versus $1 \%, p=0,02)$ et de décès $(35 \%$ 
versus $22 \%, p=0,02$ ) à trois ans, par rapport aux patients chez lesquels ces deux facteurs étaient absents. Ces deux tests permettraient donc d'identifier, avant la greffe, un groupe de patients à haut risque de développer une aspergillose invasive qui pourraient bénéficier de mesures prophylactiques spécifiques. Bien que ces hypothèses doivent être vérifiées dans des études prospectives, elles ouvrent la voie à une prise en charge personnalisée du risque infectieux dans des circonstances à haut risque. La découverte d'autres associations génétiques permettra d'affiner cette prédiction. D'autres études suggèrent en effet que le risque d'aspergillose invasive pourrait être également influencé par des polymorphismes de TLRI, TLR6, IL(interleukine)-10 ou le récepteur 2 du TNF- $\alpha$ (tumor necrosis factor) [17-20] et le risque d'aspergillose non invasive à des polymorphisme du gène $M B L$ (une association qui semble concordante dans deux petites études $[21,22]$ ) et à TLR9 [15] (Tableau I).

\section{Conclusion}

L'aspergillose invasive, par sa mortalité et son incidence relativement élevées dans un sous-groupe de patients bien déterminé, représente un exemple typique d'infection pour laquelle une approche préventive aurait un impact déterminant sur la survie. L'identification de profils génétiques à risque, indépendants de facteurs propres à la maladie et à sa présentation clinique, pourrait contribuer à améliorer la prise en charge de cette complication particulièrement redoutée, au même titre que le développement de nouveaux outils diagnostiques et thérapeutiques. $\diamond$
Preventing invasive

aspergillosis in hematopoietic

stem cells transplant recipients

\section{REMERCIEMENTS}

Les recherches de Pierre-yves Bochud sont soutenues par la Fondation Leenaards.

\section{CONFLIT D'INTÉRêTS}

Les auteurs déclarent n'avoir aucun conflit d'intérêts concernant les données publiées dans cet article.

\section{RÉFÉRENCES}

1. Bochud Py, Bochud M, Telenti A, Calandra T. Innate immunogenetics : a tool for exploring new frontiers of host defence. Lancet Infect Dis 2007 ; 7 : 531-42.

2. Bellocchio S, Moretti S, Perruccio K, et al. TLRs govern neutrophil activity in aspergillosis. J Immunol 2004 ; $173: 7406-15$.

3. Braedel S, Radsak M, Einsele H, et al. Aspergillus fumigatus antigens activate innate immune cells via toll-like receptors 2 and 4. BrJ Haematol 2004 ; 125 : 392-9.

4. Meier A, Kirschning CJ, Nikolaus T, et al. Toll-like receptor (TLR) 2 and TLR4 are essential for Aspergillusinduced activation of murine macrophages. Cell Microbiol $2003 ; 5$ : 561-70.

5. Steele C, Rapaka RR, Metz A, et al. The beta-glucan receptor dectin-1 recognizes specific morphologies of Aspergillus fumigatus. PLoS Pathog 2005; 1 : e42.

6. Dubourdeau M, Athman R, Balloy V, et al. Aspergillus fumigatus induces innate immune responses in alveolar macrophages through the MAPK pathway independently of TLR2 and TLR4. J Immunol 2006; 177 : 3994-4001.

7. Dumestre-Perard C, Lamy B, Aldebert D, et al. Aspergillus conidia activate the complement by the mannan-binding lectin $\mathrm{C} 2$ bypass mechanism. $J$ Immunol 2008; $181: 7100-5$.

8. Garlanda C, Hirsch $\varepsilon$, Bozza S, et al. Non-redundant role of the long pentraxin PTX3 in anti-fungal innate immune response. Nature $2002 ; 420$ : 182-6.

9. Madan T, Eggleton P, Kishore U, et al. Binding of pulmonary surfactant proteins $A$ and $D$ to Aspergillus fumigatus conidia enhances phagocytosis and killing by human neutrophils and alveolar macrophages. Infect Immun 1997 ; 65 : 3171-9.

10. Netea MG, Warris A, van der Meer JW, et al. Aspergillus fumigatus evades immune recognition during germination through loss of toll-like receptor-4-mediated signal transduction. J Infect Dis 2003;

$188: 320-6$.

11. Balloy V, Huerre M, Latge JP, Chignard M. Differences in patterns of infection and inflammation for corticosteroid treatment and chemotherapy in experimental invasive pulmonary aspergillosis. Infect Immun 2005 ; 73 : 494-503.

12. Quintana-Murci L, Alcais A, Abel L, Casanova JL. Immunology in natura : clinical, epidemiological and evolutionary genetics of infectious diseases. Nat Immunol $2007 ; 8$ : 1165-71.

13. Misch EA, Hawn TR. Toll-like receptor polymorphisms and susceptibility to human disease. Clin Sci (Lond) 2008; $114: 347-60$.

14. Ferwerda B, McCall MB, Verheijen $K$, et al. Functional consequences of toll-like receptor 4 polymorphisms. Mol Med 2008; $14: 346-52$.

15. Carvalho A, Pasqualotto AC, Pitzurra L, et al. Polymorphisms in toll-like receptor genes and susceptibility to pulmonary aspergillosis. J Infect Dis 2008 ; 197 : 618-21.

16. Bochud Py, Chien JW, Marr KA, et al. Toll-like receptor 4 polymorphisms and aspergillosis in stem-cell transplantation. N Engl J Med 2008 ; 359 : 1766-77.

17. Kesh S, Mensah NY, Peterlongo P, et al. TLRI and TLR6 polymorphisms are associated with susceptibility to invasive aspergillosis after allogeneic stem cell transplantation. Ann Ny Acad Sci 2005 ; 1062 : 95-103.

18. Sainz J, Hassan L, Perez $\varepsilon$, et al. Interleukin-10 promoter polymorphism as risk factor to develop invasive pulmonary aspergillosis. Immunol Lett 2007; 109: 76-82.

19. Sainz J, Perez $\varepsilon$, Hassan L, et al. Variable number of tandem repeats of TNF receptor type 2 promoter as genetic biomarker of susceptibility to develop invasive pulmonary aspergillosis. Hum Immunol 2007 ; 68: 41-50.

20. Seo KW, Kim DH, Sohn SK, et al. Protective role of interleukin-10 promoter gene polymorphism in the pathogenesis of invasive pulmonary aspergillosis after allogeneic stem cell transplantation. Bone Marrow Transplant $2005 ; 36$ : 1089-5.

21. Crosdale DJ, Poulton KV, Ollier WE, et al. Mannose-binding lectin gene polymorphisms as a susceptibility factor for chronic necrotizing pulmonary aspergillosis. J Infect Dis $2001 ; 184: 653-6$.

22. Vaid M, Kaur S, Sambatakou $H$, et al. Distinct alleles of mannose-binding lectin (MBL) and surfactant proteins $\mathrm{A}(\mathrm{SP}-\mathrm{A})$ in patients with chronic cavitary pulmonary aspergillosis and allergic bronchopulmonary aspergillosis. Clin Chem Lab Med 2007 ; 45 : 183-6.

23. Delneste $Y$, Beauvillain C, Jeannin P. Immunité naturelle structure et fonction des Toll-like receptors. Med Sci (Paris) $2007 ; 23: 67-73$.

24. Dostert C, Pétrilli V. Amiante et inflammation, rôle de I'inflammasome. Med Sci (Paris) 2008 ; 24 : 916-8.

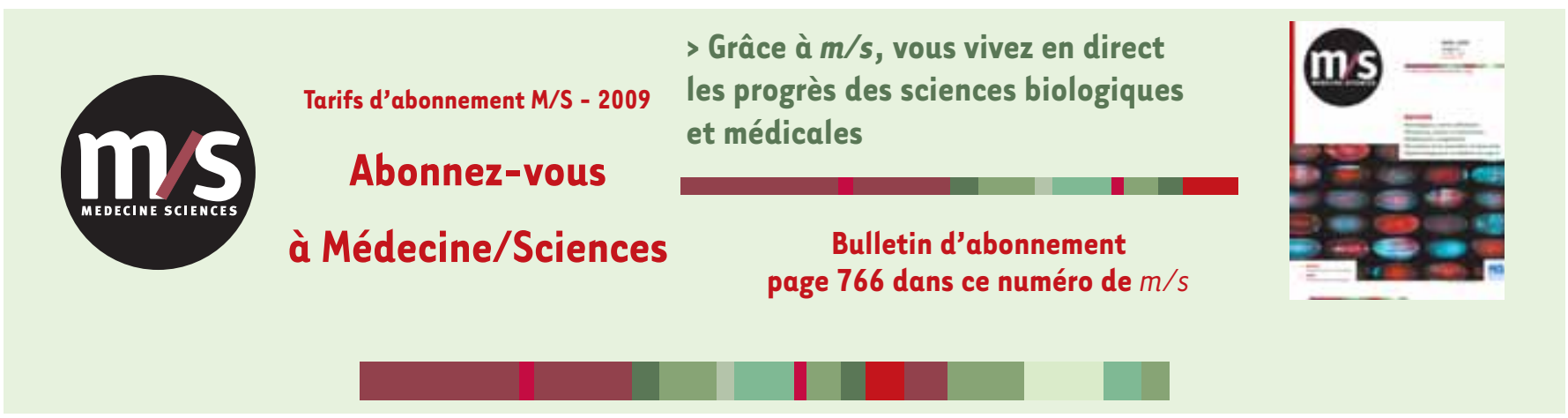

